\title{
Isocitrate lyase plays important roles in plant salt tolerance
}

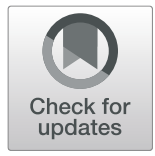

Worawat Yuenyong ${ }^{1}$, Supaart Sirikantaramas ${ }^{1,2}$, Li-Jia Qu $^{3,4}$ and Teerapong Buaboocha ${ }^{1,2^{*}}$ (D)

\begin{abstract}
Background: Isocitrate lyase (ICL) is a key enzyme in the glyoxylate cycle. In a previous study in rice, the expression of the ICL-encoding gene (Os/CL) was highly induced by salt stress and its expression was enhanced in transgenic rice lines overexpressing OsCam1-1, a calmodulin (CaM)-encoding gene. CaM has been implicated in salt tolerance mechanisms in plants; however, the cellular mechanisms mediated by CaM are not clearly understood. In this study, the role of Os/CL in plant salt tolerance mechanisms and the possible involvement of CaM were investigated using transgenic plants expressing Os/CL or OsCam 1-1.

Results: OSICL was highly expressed in senesced leaf and significantly induced by salt stress in three OsCam1-1 overexpressing transgenic rice lines as well as in wild type (WT). In WT young leaf, although OsICL expression was not affected by salt stress, all three transgenic lines exhibited highly induced expression levels. In Arabidopsis, salt stress had negative effects on germination and seedling growth of the AtICL knockout mutant (Aticl mutant). To examine the roles of OSICL we generated the following transgenic Arabidopsis lines: the Aticl mutant expressing OSICL driven by the native At/CL promoter, the Aticl mutant overexpressing OSICL driven by the 35SCaMV promoter, and WT overexpressing Os/CL driven by the 35SCaMV promoter. Under salt stress, the germination rate and seedling fresh and dry weights of the Os/CL-expressing lines were higher than those of the Aticl mutant, and the two lines with the icl mutant background were similar to the $\mathrm{WT}$. The $F_{\mathrm{v}} / F_{\mathrm{m}}$ and temperature of rosette leaves in the Os/CLexpressing lines were less affected by salt stress than they were in the Aticl mutant. Finally, glucose and fructose contents of the Aticl mutant under salt stress were highest, whereas those of Os/CL-expressing lines were similar to or lower than those of the WT.

Conclusions: $O s / C L$, a salt-responsive gene, was characterized in the transgenic Arabidopsis lines, revealing that Os/CL expression could revert the salt sensitivity phenotypes of the Aticl knockout mutant. This work provides novel evidence that supports the role of $\mathrm{ICL}$ in plant salt tolerance through the glyoxylate cycle and the possible involvement of OsCam1-1 in regulating its transcription.
\end{abstract}

Keywords: Isocitrate lyase, Calmodulin, OsCam1-1, OsICL, Salt stress

\section{Background}

Salinity is a major abiotic constraint for plants because it causes osmotic and ionic stresses. Plant responses to these stresses involve complex molecular and biochemical mechanisms. Salinity negatively affects plants in many ways, including inhibition of growth, development, photosynthesis, and yield $[1,2]$. Plants have systematic

\footnotetext{
* Correspondence: Teerapong.B@chula.ac.th

${ }^{1}$ Molecular Crop Research Unit, Department of Biochemistry, Faculty of

Science, Chulalongkorn University, Bangkok 10330, Thailand

${ }^{2}$ Omics Sciences and Bioinformatics Center, Faculty of Science,

Chulalongkorn University, Bangkok 10330, Thailand

Full list of author information is available at the end of the article
}

adaptation processes to cope with salt stress, starting with stress perception mechanisms, through signaling cascades and regulation of gene expression, to physiological responses. Previously characterized acclimation processes include osmolyte accumulation to adjust osmotic pressure in roots, morphology adaptation of roots to minimize the root surface, stomata closure to reduce water loss, elimination of excess $\mathrm{Na}^{+}$via extrusion, compartmentation and reabsorption processes, and antioxidation systems [3]. These processes require a large amount of energy from respiration, which needs to be conducted through cost-efficient strategies so the plants

(c) The Author(s). 2019 Open Access This article is distributed under the terms of the Creative Commons Attribution 4.0 International License (http://creativecommons.org/licenses/by/4.0/), which permits unrestricted use, distribution, and reproduction in any medium, provided you give appropriate credit to the original author(s) and the source, provide a link to the Creative Commons license, and indicate if changes were made. The Creative Commons Public Domain Dedication waiver (http://creativecommons.org/publicdomain/zero/1.0/) applies to the data made available in this article, unless otherwise stated. 
can maintain the ability to grow, through more slowly, and produce a harvestable yield [4].

$\mathrm{Ca}^{2+}$ has long been known as a major second messenger in signal transduction pathways of eukaryotic cell. Plants use calcium signaling to perceive and respond to environmental stimuli, including abiotic stress [5]. The calcium-signaling pathway is involved in alterations of $\mathrm{Ca}^{2+}$ concentrations in the cytosol that result from the release of $\mathrm{Ca}^{2+}$ from intracellular organelles or from outside the cell $[5,6]$. First, the membrane receptor recognizes the stress signal, resulting in activation of phospholipase $\mathrm{C}$. The activated phospholipase $\mathrm{C}$ hydrolyses phosphatidylinositol-4,5-bisphosphate to inositol-1,4, 5-trisphosphate, which mediates an increase in the concentration of cytoplasmic $\mathrm{Ca}^{2+}$. The signal is perceived by $\mathrm{Ca}^{2+}$ sensors, which enhances downstream effects via, for example, kinases or phosphatases that regulate the expression of stress responsive genes, leading to adaptive physiological responses [7, 8]. Over 250 calcium sensor proteins have been identified in Arabidopsis, and they have been categorized in three major families: calmodulin (CaM) and calmodulin-like proteins, calcineurin-B-like proteins, and calcium-dependent protein kinases and calcium- and calmodulin-dependent protein kinases [9].

Our previous transcriptomic analysis showed that the rice isocitrate lyase gene (OsICL) was induced by salt stress, and that its expression was enhanced by the combined effect of salt stress and overexpression of a rice calmodulin gene (OsCam1-1) [10]. Isocitrate lyase (ICL) is a key enzyme in the glyoxylate cycle, which is the bypassed pathway of the TCA cycle that converts isocitrate to glyoxylate and succinate. In Arabidopsis during germination, ICL plays an important role in lipid-sugar conversion using the acetyl unit from acetyl$\mathrm{CoA}$, the product of $\beta$-oxidation, via the glyoxylate cycle and gluconeogenesis $[11,12] . I C L$ is a single-copy gene in both rice [13] and Arabidopsis [14]. The nucleotide sequences of the $I C L$ genes and the encoded amino acid sequences of rice and Arabidopsis share 66.07 and $72.50 \%$ identities, respectively. Cooper and Beevers [15] studied mitochondria and glyoxysomes of castor bean endosperm and found that more than $85 \%$ of the enzyme activity in the glyoxysomes was from ICL and malate synthase, another key enzyme in the glyoxylate cycle. ICL and malate synthase were found to be involved in the transition of leaf peroxisomes to glyoxysomes, and this process was correlated with senescence [16].

Because the CaM action involves many downstream components that are spread widely in cells, the roles of $\mathrm{CaM}$ in conjunction with these components have not been completely understood, especially the roles of CaM in salt stress responses. Although $\mathrm{CaM}$ has been shown to contribute to the salt stress tolerance of plants [17, 18], the actual mechanisms have not been characterized.
Our previous study showed that OsICL expression was highly induced by OsCam1-1 overexpression [10], however, to our knowledge, there are no clear evidence showing that ICL facilitates plant salt tolerance. Therefore, the roles of OsICL and CaM in salt tolerance and $\mathrm{CaM}$ involvement were investigated in this study.

\section{Results}

\section{Induced $O s / C L$ expression in rice lines overexpressing} OsCam 1-1 under salt stress

To verify our previously reported transcriptome results of the transgenic rice overexpressing OsCam1-1 [10], transcript expression levels of OsICL in 3-week old seedling shoots of three independent transgenic lines, L1, L2, and L7, were examined by qRT-PCR. We used the $2^{-(\Delta \Delta C T)}$ method to analyze the relative changes in gene expression with the expression of OsICL in the wild type (WT) rice under non-stress condition as the baseline. The expression levels of OsICL in WT and in the three transgenic lines under the non-stress condition were not different. Under the salt stress condition, OsICL expression levels in the three transgenic lines increased sharply, and were much higher than in the WT (Fig. 1a). The OsICL expression levels increased by about 500 -fold in the WT, and by about 1300-, 1400-, and 800-folds in L1, L2, and L7, respectively, compared with the baseline.

We determined the expression levels of OsICL in WT and in the three transgenic rice lines using first leaf (lowest leaf) to represent a senesced leaf and the third leaf to represent a young leaf. Under the non-stress condition, the senesced leaves showed significantly higher OsICL expression levels than the young leaves. Under salt stress condition, the OsICL expression levels increased in both leaves, but the senesced leaves still showed higher OsICL expression levels than the young leaves (Fig. 1b, c). In WT, L1, L2, and L7, the fold changes in senesced leaves were about 340, 390, 435, and 800 over the baseline of the WT young leaf under the non-stress condition. However, when compared with the WT senesced leaf under salt stress these changes were statistically significant only for L7. Interestingly, the young leaves from the three transgenic lines showed increased OsICL expression levels with fold changes in L1, L2, and L7 of about 40, 25, and 15 over the baseline, whereas there was no such change in the young leaf from WT (Fig. 1b).

To examine the promoter sequence of OsICL, we used the 2020-bp upstream sequence, which included a predicted 5'UTR of $248 \mathrm{bp}$ from the Phytozome database [19] and analyzed it using PLACE, a web-based tool for analyzing cis-acting regulatory DNA elements [20]. Nine OsWRKY71 (LOC_Os02g08440) binding regions were predicted upstream of the 5'UTR and one OsWRKY71 binding region was predicted in the 5'UTR. In addition, 

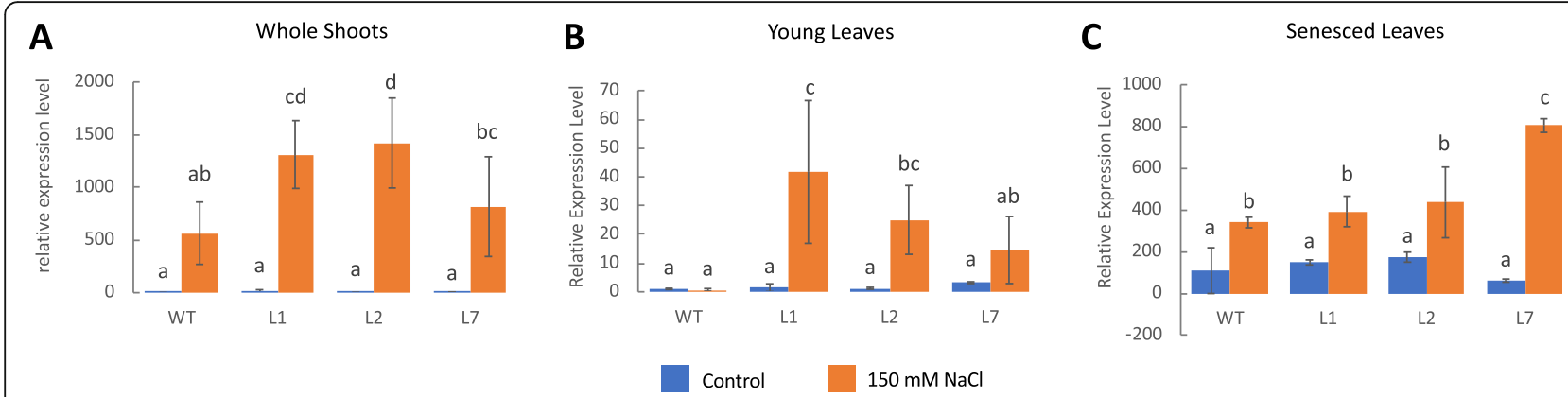

Senesced Leaves

Fig. 1 a qRT-PCR analysis showing Os/CL gene expression levels in the whole shoots, $\mathbf{b}$ young leaves and $\mathbf{c}$ senesced leaves of the three transgenic rice over-expressing OsCam 1-1 (L1, L2, L7) and WT under non-stress and salt stress at $150 \mathrm{mM} \mathrm{NaCl}$ for $4 \mathrm{~h}(\mathrm{~N}=4)$. The relative expression of the whole shoots was compared using whole shoots of WT under non-stress as base line, and the relative expression level of the young and senesced leaves was compared using young leaves under non-stress as baseline. One-way ANOVA with Duncan multiple range test with criteria of $p<0.05$ was separately applied for statistical data analysis of whole shoots, young leaves, and senesced leaves. Data are shown as the mean $\pm 1 S D$, and means with the same letter are not significantly different

two abscisic acid (ABA) responsive elements (ABRE) were found upstream of the 5'UTR (Fig. 2).

\section{Generation and verification of transgenic Arabidopsis expressing OsICL}

Three transgenic Arabidopsis lines expressing OsICL were generated successfully: 3FL9, the Aticl Arabidopsis mutant expressing OsICL driven by the 2138-bp upstream sequence of the AtICL gene; OXOsICL/icl, the Aticl mutant expressing OsICL driven by the 35SCaMV promoter; and OXOsICL/WT, WT Arabidopsis expressing OsICL driven by the 35SCaMV promoter. PCR and agarose gel electrophoresis confirmed construction of the recombinant plasmids (Additional file $1 \mathrm{~A}$ and $\mathrm{B}$ ) and the background genotypes of all five plants examined: 3FL9, OXOsICL/icl, OXOsICL/WT, Aticl mutant, and WT (Additional file 1C) and the OsICL gene insertion in all OsICL-expressing transgenic Arabidopsis lines (Additional file 1D).

The qRT-PCR analysis showed that OsICL was expressed in the three transgenic Arabidopsis containing the gene construct but not in the WT and Aticl mutant under both non-stress and salt stress conditions (Table 1). Among the three transgenic Arabidopsis lines, OXOsICL/ WT exhibited outstandingly high OsICL expression levels under both the non-stress and salt stress conditions; however, the OsICL expression level was significantly higher under salt stress than under the non-stress condition. For the two transgenic Arabidopsis lines, 3FL9 and OXOsICL/ $\mathrm{icl}$, with the Aticl mutant background, the OsICL expression levels were lower than they were for OXOsICL/WT, but its OsICL expression levels were higher for OXOsICL/ $i c l$ than for 3FL9. Expression of the native Arabidopsis isocitrate lyase gene (AtICL) was also determined, and the results showed that, under salt stress, AtICL expression was detected only in WT and OXOsICL/WT, the two lines with the WT background (Table 2).

The activity of ICL was determined using an assay that measured the amount of glyoxylate production. The highest ICL activity was found in OXOsICL/WT under both the non-stress and salt stress conditions (Fig. 3). Under the non-stress condition, ICL activity was higher in 3FL9 and OXOsICL/icl than the WT and Aticl mutant, however, under salt stress, ICL activity increased in WT but not in the Aticl mutant and other lines with the Aticl mutant background.

\section{Transgenic Arabidopsis expressing OsICL exhibited better germination and seedling growth under salt stress than the Aticl mutant}

Under the non-stress condition, all Arabidopsis lines almost completely germinated after 1 day (Fig. 4). Under $120 \mathrm{mM} \mathrm{NaCl}$ stress condition, WT and 3FL9 had the

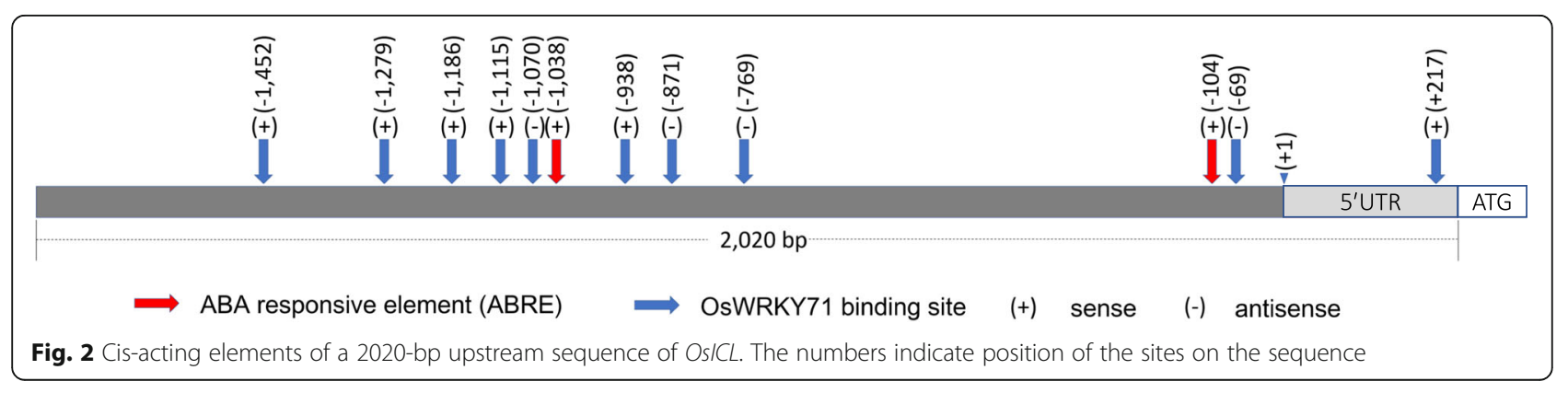


Table 1 Expression levels obtained by qRT-PCR of Os/CL in 10day-old transgenic Arabidopsis seedlings

\begin{tabular}{lll}
\hline Lines & $2^{-(\Delta C \mathrm{C})} \pm \mathrm{SD}$ & \\
\cline { 2 - 3 } & Control & $120 \mathrm{mM} \mathrm{NaCl}$ \\
\hline wild type & $\mathrm{ND}$ & $\mathrm{ND}$ \\
Aticl mutant & $\mathrm{ND}$ & $\mathrm{ND}$ \\
3 FL9 & $0.0247 \pm 0.0040^{\mathrm{a}}$ & $0.0077 \pm 0.0030^{\mathrm{a}}$ \\
OXOs/CL/icl & $0.1251 \pm 0.0391^{\mathrm{a}}$ & $0.0745 \pm 0.0194^{\mathrm{a}}$ \\
OXOSICL/WT & $3.6645 \pm 0.6099^{\mathrm{b}}$ & $5.2608 \pm 0.9758^{\mathrm{c}}$ \\
\hline
\end{tabular}

One-way ANOVA with Duncan multiple range test was used for the data analysis

Data are shown as the mean $\pm 1 S D$, and different lowercase letters indicate significant difference among plant lines under both control and stress conditions $(p<0.05)$

ND, no OsICL expression was detected

highest germination rates whereas the Aticl Arabidopsis mutant had the lowest germination rate. Interestingly, although the two transgenic Arabidopsis lines overexpressing OsICL under the control of the 35SCaMV promoter, OXOsICL/WT and OXOsICL/icl, had lower germination rate than the WT and 3FL9, they had higher germination rates under salt stress than the Aticl mutant, and this was more pronounced at higher $\mathrm{NaCl}$ concentrations.

To investigate the effect of OsICL expression levels on seedling growth under salt stress, the fresh and dry weights of the five Arabidopsis lines were determined. The results showed that under the non-stress condition the fresh weights and dry weights of the five Arabidopsis lines were not significantly different. Under salt-stress, the changes in the fresh and dry weights had a similar pattern, in which the Aticl mutant had significantly lower fresh and dry weighs than the other Arabidopsis lines (Fig. 5). Furthermore, by appearance, the Aticl mutant showed growth defect more frequently than the other Arabidopsis lines under salt stress, particularly under high salt concentration (Fig. 6).

Table 2 Expression levels obtained by qRT-PCR of At/CL in 10day-old Arabidopsis seedlings

\begin{tabular}{lll}
\hline Lines & \multicolumn{2}{c}{$2^{-(\Delta C T)} \pm S D$} \\
\cline { 2 - 3 } & Control & $120 \mathrm{mM} \mathrm{NaCl}$ \\
\hline wild type & $\mathrm{ND}$ & $0.0027 \pm 0.0015^{\mathrm{b}}$ \\
Aticl mutant & $\mathrm{ND}$ & $\mathrm{ND}$ \\
3 FL9 & $\mathrm{ND}$ & $\mathrm{ND}$ \\
OXOs/CL/iCl & $\mathrm{ND}$ & $\mathrm{ND}$ \\
OXOSICL/WT & $\mathrm{ND}$ & $0.0003 \pm 0.0002^{\mathrm{a}}$ \\
\hline
\end{tabular}

One-way ANOVA with Duncan multiple range test was used for the data analysis

Data are shown as the mean $\pm 1 \mathrm{SD}$, and different lowercase letters indicate significant difference $(p<0.05)$

ND, no At/CL expression was detected
Transgenic Arabidopsis expressing OsICL exhibited less salt stress effects than the Aticl mutant

To examine the effect of salt stress on photosynthesis, $F_{\mathrm{v}} / F_{\mathrm{m}}$, which indicates maximum potential quantum efficiency of Photosystem II in the dark-adapted state [21], was measured using a pocket chlorophyll fluorimeter. The results showed that under the non-stress condition there were no difference in $F_{\mathrm{v}} / F_{\mathrm{m}}$ in the five Arabidopsis lines (Fig. 7a). Under salt stress, the $F_{\mathrm{v}} / F_{\mathrm{m}}$ decreased significantly in the five Arabidopsis lines; the Aticl mutant had the lowest $F_{\mathrm{v}} / F_{\mathrm{m}}$ values and OXOsICL/WT had the highest. Rosette leaf temperature of 4-week-old transgenic Arabidopsis lines expressing OsICL, WT, and the Aticl mutant was measured after non-stress and $300 \mathrm{mM}$ $\mathrm{NaCl}$ stress for 3 days. Under the non-stress condition, no differences were detected in the temperatures in the five Arabidopsis lines (Fig. $7 \mathrm{~b}$ and Additional file 2). Under salt stress, the temperature increased in all five lines, especially in the Aticl mutant, which had highest rosette leaf temperature. Overall, the changes in $F_{\mathrm{v}} / F_{\mathrm{m}}$ and rosette leaf temperature indicated that the Aticl mutant was the most affected by salt stress among the five Arabidopsis lines examined and OsICL expression reverted these salt sensitivity phenotypes of the Aticl knockout mutant.

\section{Effect on sugar content of OsICL expression in Arabidopsis under salt stress}

The role of ICL in the plant glyoxylate cycle involves the bypass of the TCA and the conversion of lipid to sugar via a gluconeogenesis process, so we determined the sucrose, glucose, and fructose contents in cauline and rosette leaves of 4-week-old transgenic Arabidopsis lines expressing OsICL, Aticl mutant, and WT, under nonstress and $300 \mathrm{mM} \mathrm{NaCl}$ stress. We found that the sucrose content of cauline leaf but not rosette leaf increased under salt stress in all five Arabidopsis lines (Fig. 8a, b), whereas, the glucose and fructose contents increased significantly in both cauline and rosette leaves under the same salt stress condition (Fig. 8c-f). Surprisingly, the glucose and fructose contents of the cauline and rosette leaves in the Aticl mutant under salt stress were one of the highest comparing among the five Arabidopsis lines. In cauline leaf, the glucose and fructose contents of the OsICL-expressing Arabidopsis lines, especially OXOsICL/WT, under salt stress were noticeably lower than the content of the Aticl mutant, which was similar to that of the WT (Fig. 8c, e). Interestingly in rosette leaf, the glucose and fructose contents of the Aticl mutant under salt stress were significantly higher than those of the WT, whereas the glucose and fructose contents of the OsICL-expressing Arabidopsis lines were more varied and similar to those of the WT (Fig. 8d, f). 


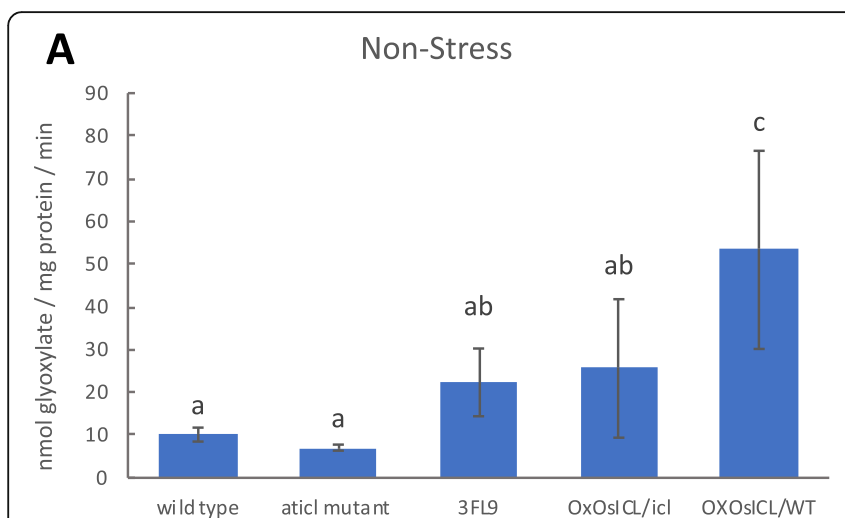

B

$120 \mathrm{mM} \mathrm{NaCl}$

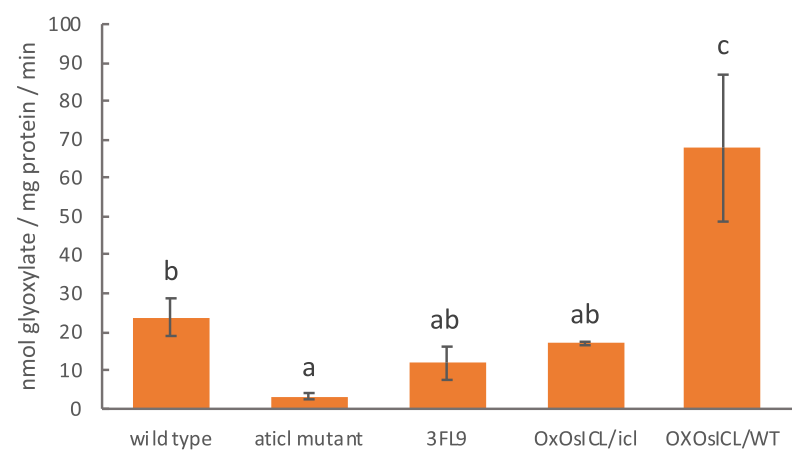

Fig. 3 Isocitrate lyase activity of the five Arabidopsis lines $\mathbf{a}$ under normal condition, and $\mathbf{b}$ under salt stress at $120 \mathrm{mM} \mathrm{NaCl}$ for 10 days $(\mathrm{N}=3)$. One-way ANOVA with Duncan multiple range test $(p<0.05)$ was used in the isocitrate lyase activity data analysis. Data are shown as the mean \pm $1 \mathrm{SD}$, and means with the same letter are not significantly different

\section{Discussion}

The transgenic rice overexpressing OsCam1-1, which exhibited higher salt stress tolerance than the WT [18], was shown to have highly increased OsICL transcript levels under salt stress, in contrast with Pinus pinea seeds, in which ICL activity was decreased under salt stress [22]. This suggested that, in rice, ICL may play important roles in salt tolerance mechanisms. Senescence is known to affect the expression levels of ICL genes [23, 24], and we found that OsICL was highly expressed in senesced leaf compared with young leaf under the non-stress condition, which is likely under a pre-programmed developmental process. In previous reports, ICL was also found to be involved in senescence. For example, Gut and Matile [25] found that ICL gene expression was induced in senesced barley leaves, and McLaughlin and Smith [26] found that ICL gene expression was induced by acetate under dark condition. In barley leaf and cucumber cotyledon, the abundance of ICL protein increased in the absence of sucrose during darkness-induced senescence in which chlorophyll and protein contents decreased, whereas its abundance was decreased by sucrose supplementation, indicating that ICL and the glyoxylate cycle function in carbohydrate-starved tissues during senescence [27]. The authors suggested that ICL may be involved in the conversion of lipids to organic acids, which are then used in the

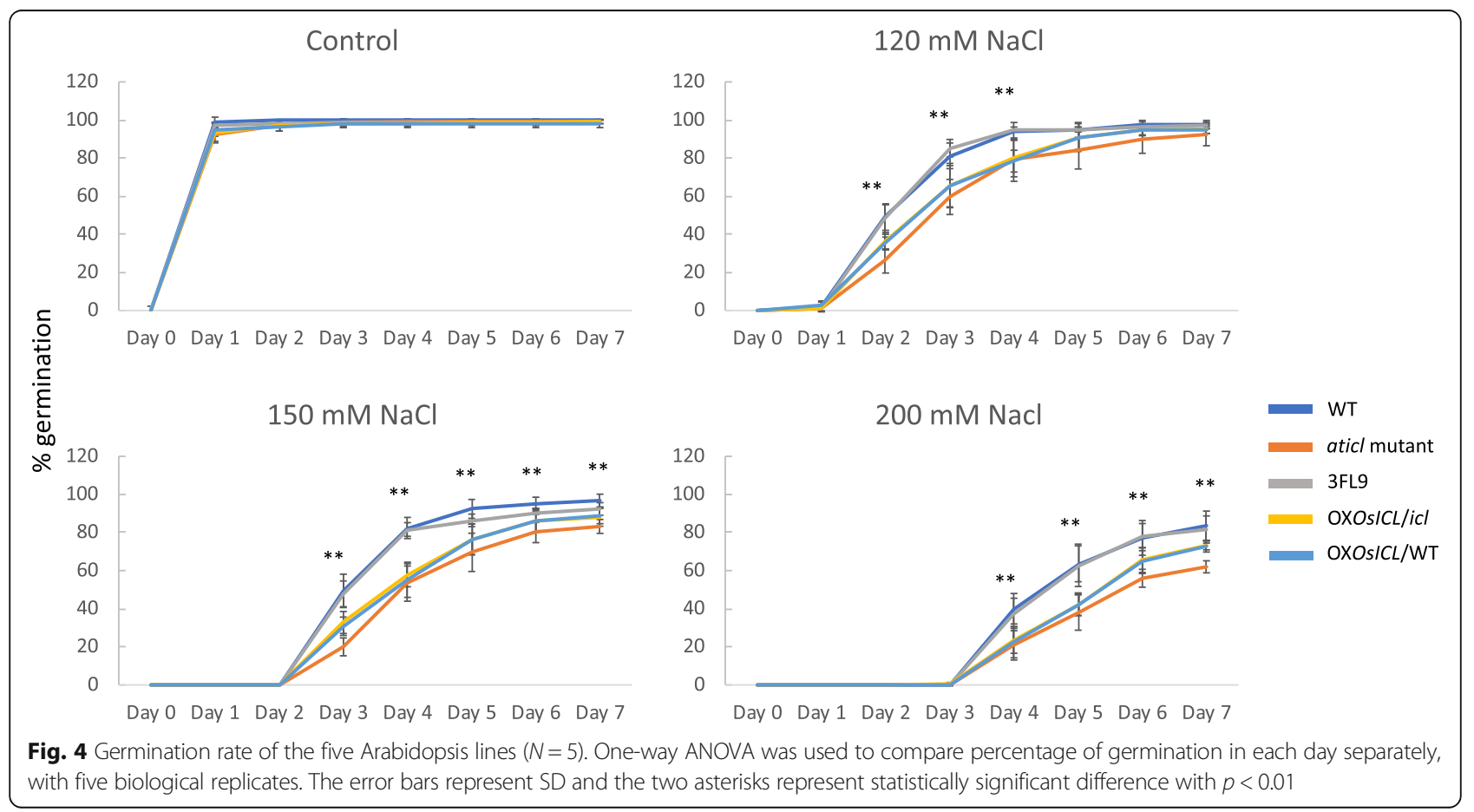




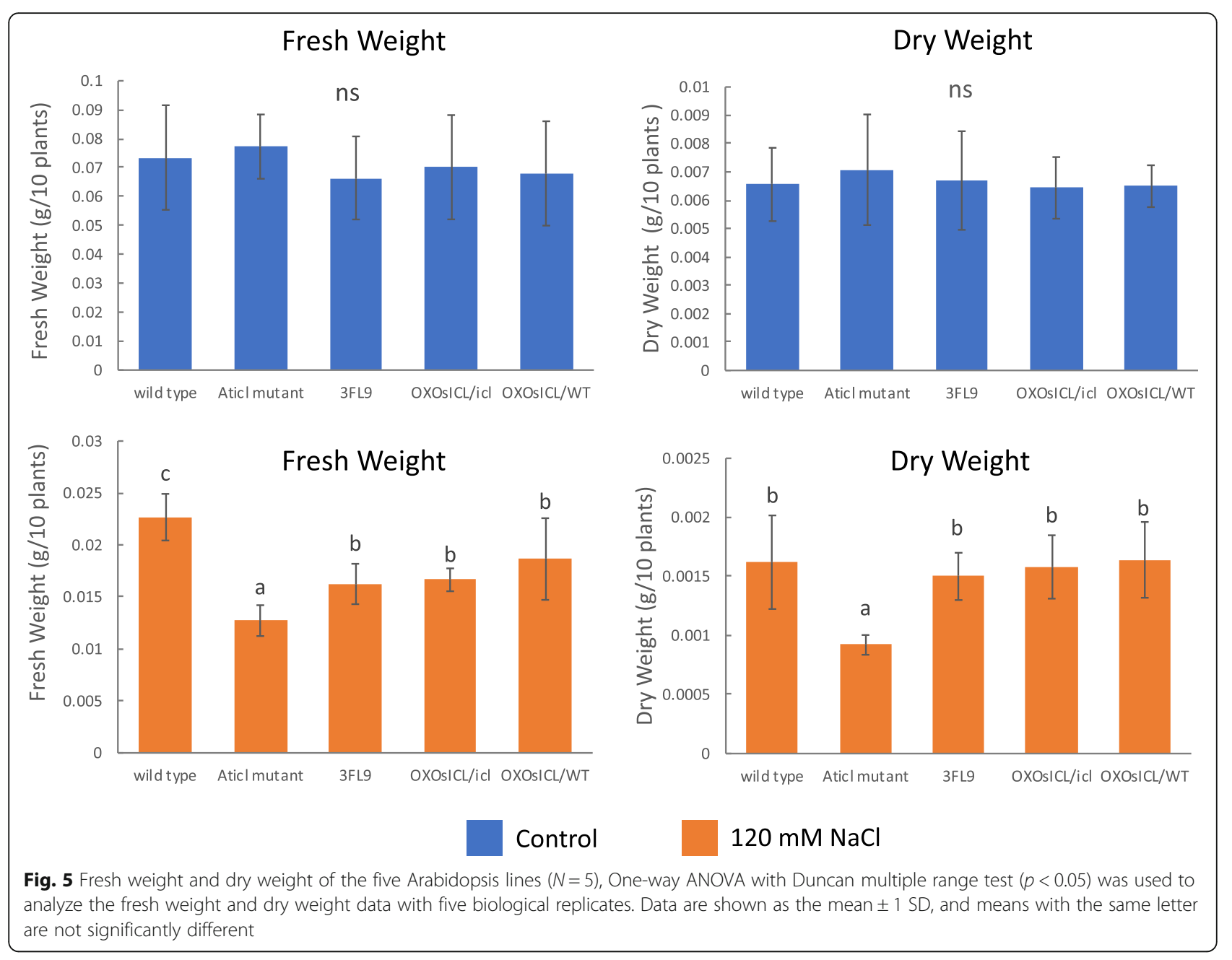

mobilization of amino acids from leaf proteins. The increase in OsICL expression under salt stress that we observed may trigger similar metabolic adjustments during the period of reduced carbon availability.

We found that OsICL transcript levels increased in senesced leaf of WT under salt stress, but not in young leaf. Some studies have reported that salt stress can induce plant senescence. For example, Munns [28] reported that toxicity of $\mathrm{Na}^{+}$caused by salt stress resulted in premature senescence, and Lutts et al. [29] found that $\mathrm{NaCl}$ affected senescence-related parameters in rice leaf, including chlorophyll fluorescence, membrane permeability, and protein and chlorophyll concentrations. A previous report suggested that CaM1 positively controlled Arabidopsis leaf senescence as leaf yellowing, reactive oxygen species (ROS) accumulation, and expression of SAG12, a senescence-associated gene, were enhanced in Arabidopsis overexpressing CaM1 [30]. Although we found that the OsICL upregulation by salt stress in the senesced leaf of the OsCam1-1-overexpressing lines was not clearly different from that of the WT, possibly because of their already high expression levels, the OsICL expression level was highly up-regulated in the young leaf of the OsCam1-1 overexpressing lines, but not in the WT. These results suggest that the highly up-regulated OsICL expression in young leaf caused by the OsCam1-1 overexpression may contribute to the salt tolerance phenotype.

The transgenic rice overexpressing OsCam1-1 reported previously exhibited higher ABA content than the WT under salt stress [18]. The presence of two putative ABREs in the 2-kb upstream sequence of OsICL suggests that OsICL expression under salt stress was induced by ABA. ABA is a stress hormone in plants that is involved in changes of long-distance transport, stomatal behavior, and gas exchange [31]. ABA controls the transcription of target genes via regulating interaction of transcription factors, including DREB2A/2B, AREB1, and RD22BP1 and $\mathrm{MYC} / \mathrm{MYB}$, with their corresponding cis-acting elements, DRE/CRT, ABRE, and MYCRS/MYBRS, respectively [32]. ABA also promotes senescence [33]. Furthermore, in our previous transcriptome study we found that OsWRKY71 (LOC_Os02g08440) expression was induced and enhanced 


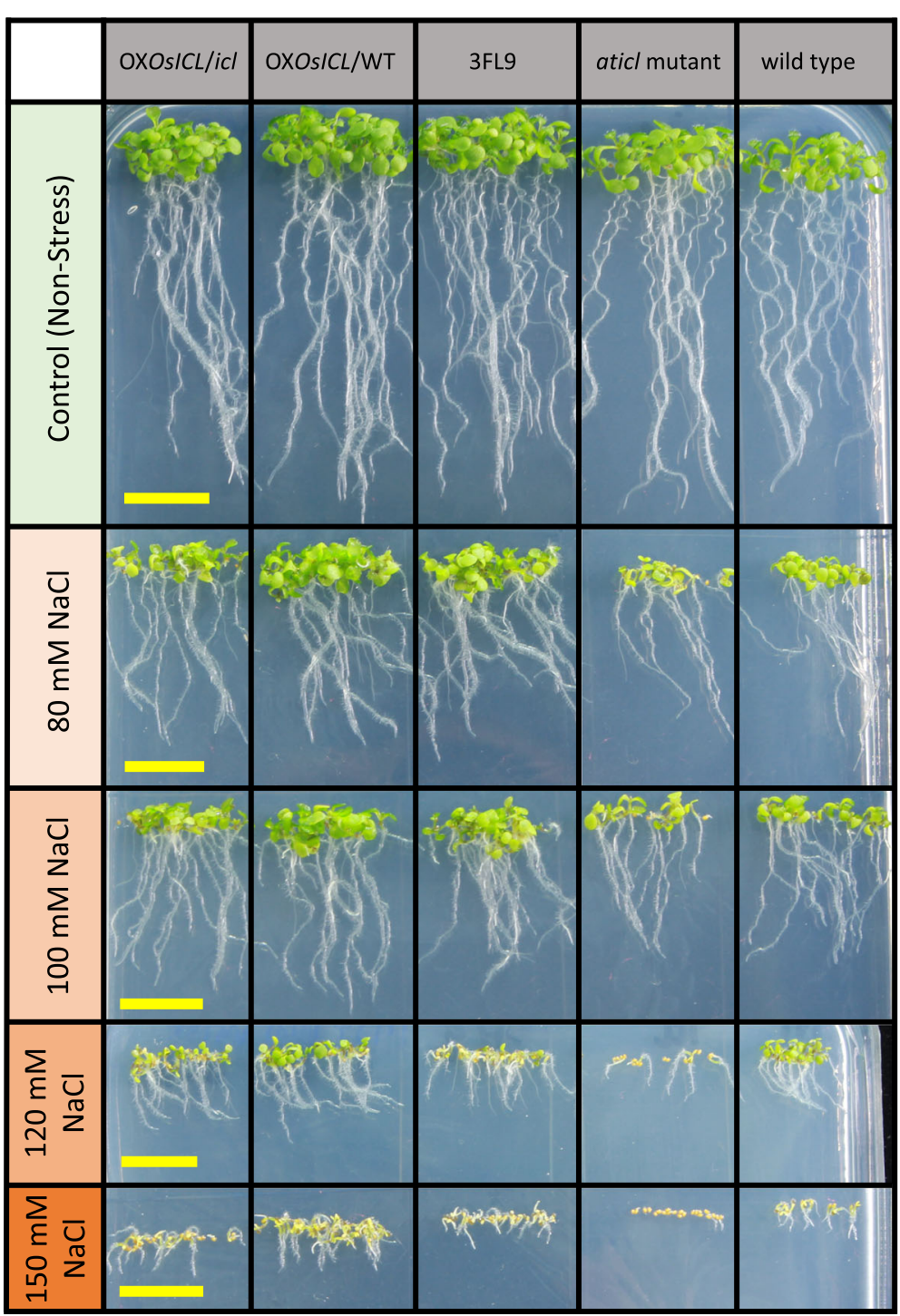

Fig. 6 The five Arabidopsis lines growing in MS medium containing various $\mathrm{NaCl}$ concentrations at day 10 of growth. The scale bars represent $1 \mathrm{~cm}$

by OsCam1-1 overexpression [10], which could in turn up-regulate the expression of OsICL. This finding supports the presence of the nine putative OsWRKY71 binding regions that were detected in the 2-kb upstream sequence of OSICL. Another report in rice aleurone cells showed that OsWRKY71 was induced by ABA [34]. Together, these results indicate that transcription of OSICL may be directly driven by the OsWRKY71 transcription factor, which is influenced by salt-stress-induced senescence, ABA, and OsCam1-1 expression.

The growth parameters, namely, germination rate, and fresh and dry weights at the seedling stage of the three transgenic OsICL-expressing Arabidopsis lines compared with the Aticl mutant and the WT showed the impact of OsICL activity on plant growth under salt stress. The highest degree of growth inhibition under salt stress observed in the Aticl mutant suggests that ICL affects salt stress tolerance in the germination growth stage. Arabidopsis is an oil seed plant [35], therefore during germination, stored lipid in the seed needs to be converted to an available carbon source for generating energy. Previous studies showed that the icl mutant Arabidopsis seedling had defective growth in a non-sucrose supplemented medium, especially under the dark condition, and interestingly, when $\left[{ }^{14} \mathrm{C}\right]$ acetate was supplied to the $i c l$ mutant line, the amount of ${ }^{14} \mathrm{C}$-labeled sugar in the $i c l$ mutant line was significantly lower than the WT $[11,12]$. This suggests that ICL may play a role during germination by using the acetyl unit from acetyl-CoA, the product of stored lipid degradation by $\beta$-oxidation, to synthesize sugars via the glyoxylate cycle and gluconeogenesis. A study of two sugar beet hybrids showed that the high vigor hybrid had 


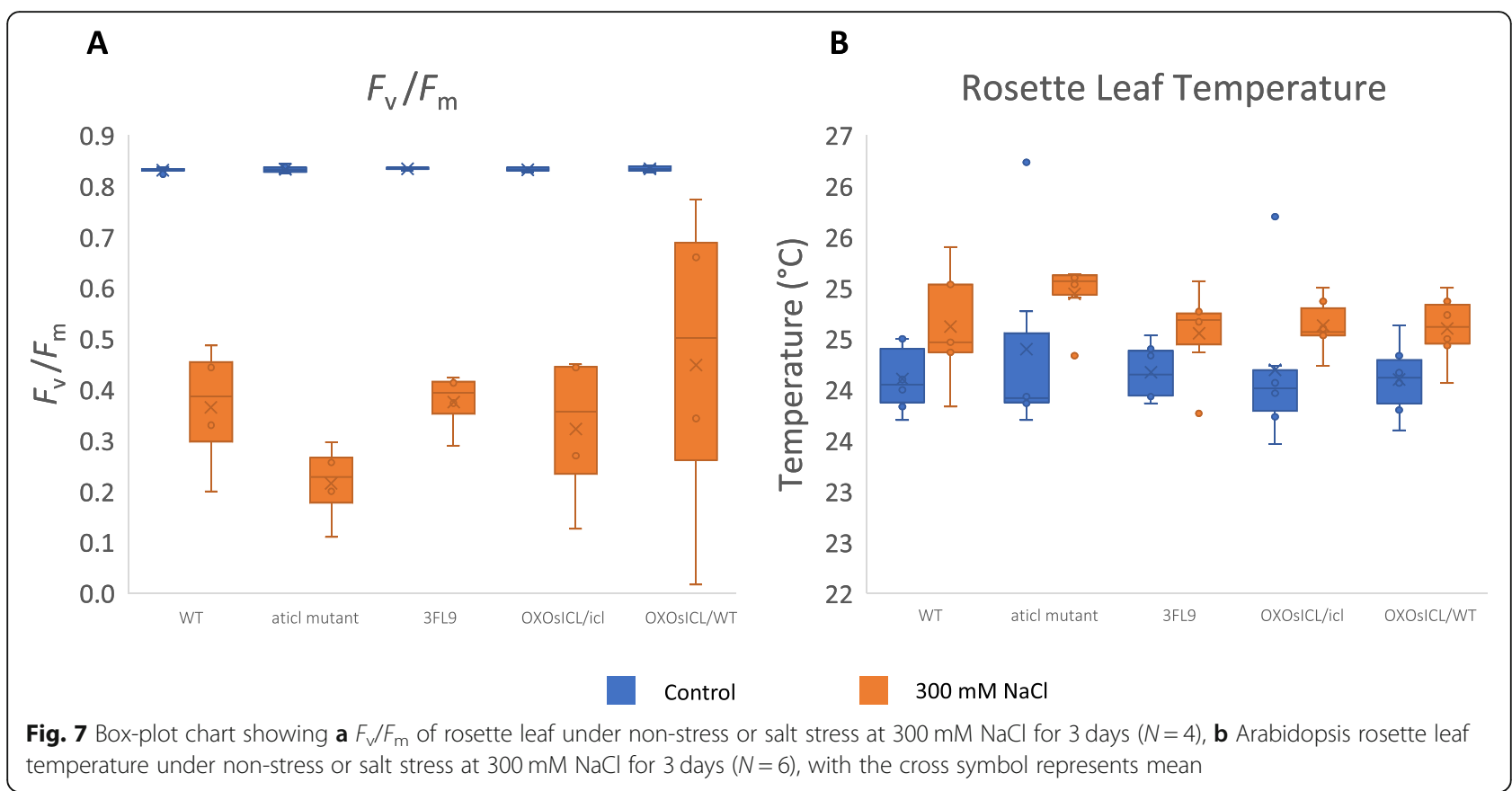

higher ICL transcript levels and higher ICL activity than the low vigor hybrid, especially under salt and $\mathrm{H}_{2} \mathrm{O}_{2}$ stresses, so it was suggested that ICL was a marker for seedling vigor [36]. Together, these results suggest that ICL might play multifunctional roles including salt tolerance, in the plants by modulating energy metabolism.

The results for the vegetative stage further indicate that salt stress affected the OsICL-expressing lines to a lesser degree, as indicated by the higher $F_{\mathrm{v}} / F_{\mathrm{m}}$ values and lower temperature in the leaf, compared with the Aticl mutant. Under salt stress, the rosette leaf temperature of the Aticl mutant was highest and $F_{\mathrm{v}} / F_{\mathrm{m}}$ was lowest among the plants examined, which indicated that photosystem II of the Aticl mutant was more disrupted. It has been reported that rice leaf temperature measured by infrared thermal imaging was higher when the plant was exposed to high salt concentrations, and the temperature was negatively correlated to stomatal conductance and relative water content [37]. This suggests that the Aticl mutant was most affected by salt stress and OsICL expression reverted the salt sensitivity phenotype of the Aticl mutant. This idea is supported by the phenotype of the OsICL-overexpressing line on the WT background, which had the least affected $F_{\mathrm{v}} / F_{\mathrm{m}}$ value under salt stress.

Figure 9 describes a proposed salt-responsive regulatory mechanism of ICL that can shift energy metabolism from the TCA cycle to the glyoxylate cycle during salt stress. In the glyoxylate cycle, ICL generates succinate and glyoxylate, which is the precursor for malate. The catalysis of succinate to fumarate generates one $\mathrm{FADH}_{2}$ and the catalysis of malate to oxaloacetate generates one
NADH. The glyoxylate cycle bypasses the two decarboxylation steps of the TCA cycle. Two steps of the glyoxylate cycle use the acetyl unit from acetyl-CoA, namely, the conversion of glyoxylate to malate and the subsequent conversion of oxaloacetate to citrate. AcetylCoA can be generated by $\beta$-oxidation or catabolism of some amino acids, namely, leucine, isoleucine, valine, alanine, serine, and cysteine [38]. A previous report in wheat revealed that the TCA cycle, which is a major respiratory process, was inhibited by salt stress and that the pyruvate dehydrogenase and 2-oxoglutarate dehydrogenase complexes were salt sensitive; therefore, the respiratory metabolism was shifted to the GABA shunt pathway to provide an alternative carbon source [39]. In our previous study in rice, we found that the expression levels of genes encoding aconitase and malate synthase were up-regulated by the effect of salt stress and OsCam1-1 overexpression [10]. Because the pyruvate dehydrogenase complex was sensitive to salt stress, we speculated that pyruvate might instead be fluxed to pyruvate carboxylase to generate oxaloacetate. Additionally, 2-oxoglutarate dehydrogenase complex might be inhibited under salt stress. Therefore, we propose that the disrupted TCA cycle could lead to metabolite flux into the glyoxylate cycle to bypass the salt-inhibited enzymes.

The glyoxylate cycle generates NADH and $\mathrm{FADH}_{2}$ to be fed into the electron transport chain to generate energy. Therefore, lacking the important activity of ICL would disrupt the metabolic flux of the glyoxylate cycle as mentioned above, and with the inhibition of pyruvate dehydrogenase and 2-oxoglutarate dehydrogenase under 


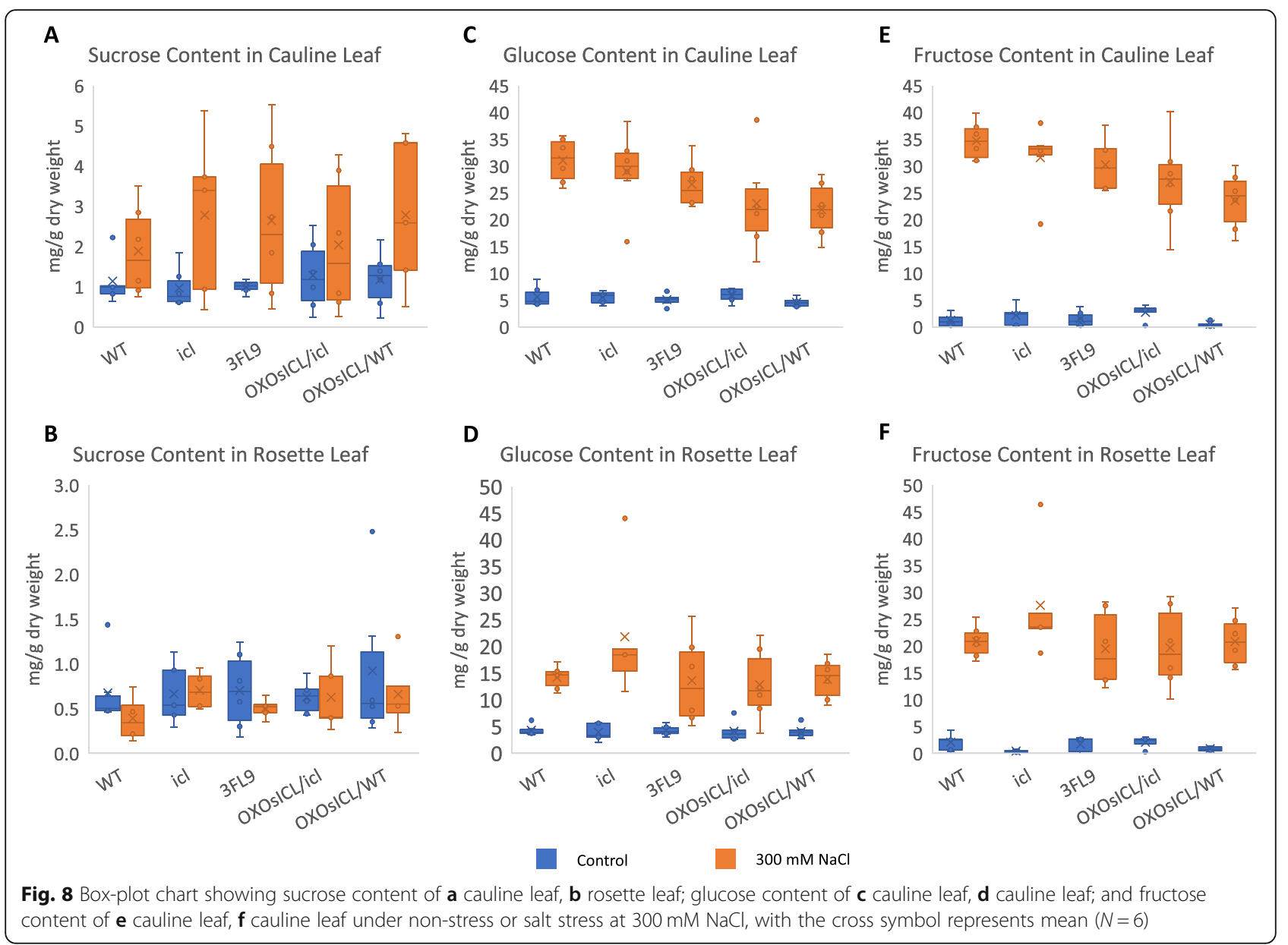

salt stress, this would presumably result in glucose and fructose being inefficiently fed into the metabolic flux of the central energy metabolism process. We found that the glucose and fructose contents of the Aticl mutant, which was the most salt-sensitive line, were highest among the five Arabidopsis plants examined, and the OsICL expression reverted it. Together, these results provide novel evidence to support the role of ICL, the key enzyme in the glyoxylate cycle, in plant salt tolerance.

\section{Conclusions}

This study suggests that, in rice, OsICL plays roles under salt stress through acclimation of energy metabolism and that OsCam1-1 may be involved in regulating its transcription. The role of OsICL, a salt-responsive gene was characterized in transgenic Arabidopsis revealing that ICL expression reverted the salt sensitivity phenotypes as well as the glucose and fructose contents of the Aticl mutant. Together, these results suggest that ICL may facilitate the shift of energy metabolism to the glyoxylate cycle to modulate carbon balance and provide necessary energy during salinity stress, thereby contributing to salt tolerance in plants.

\section{Methods}

Plant materials and growing conditions

Three lines of transgenic Khao Dawk Mali 105 (salt-sensitive) rice overexpressing OsCam1-1 [18] were grown hydroponically in Yoshida solution [40] for 3 weeks with completely randomized design. Then, the rice was treated with $150 \mathrm{mM} \mathrm{NaCl}$ for $4 \mathrm{~h}$. The seedlings were collected and snap frozen in liquid nitrogen. Seeds of the Aticl Arabidopsis mutant (GK-008E03) were obtained from Nottingham Arabidopsis Stock Centre (UK). The seed was decontaminated by quickly rinsing with $75 \%$ ethanol and soaking in $2 \% \mathrm{NaOCl}$ for 10 min before rinsing with sterilized water containing Tween 80 for 58 times, and then transferred to a Murashige \& Skoog (MS) medium (PhytoTechnology Laboratories ${ }^{\circ}$, USA) plate with $1 \% \mathrm{w} / \mathrm{v}$ sucrose. The seed was sown on the plate and stored at $4{ }^{\circ} \mathrm{C}$ for 2 days. Then the plate was moved to a growth chamber or a growth room at $25^{\circ} \mathrm{C}$ under $16-\mathrm{h} / 8$-h light/dark period for 1 week. The plant 


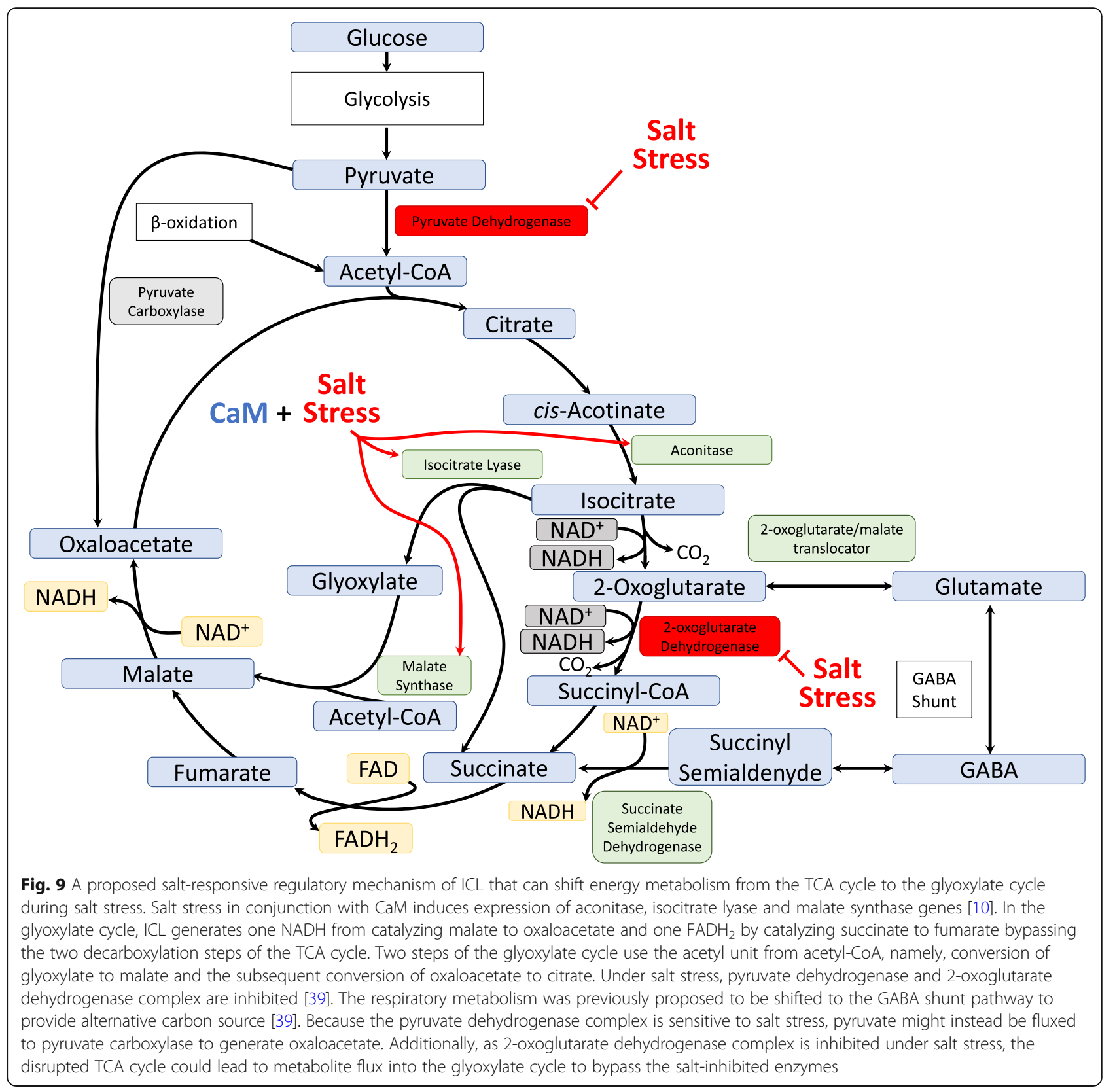

was transferred to a pot containing planting material, peat moss:perlite:vermiculite in a 3:1:1 ratio, and grown under the same condition, and watered every 2-3 days until the plant matured. The seeds were harvested and stored at $4{ }^{\circ} \mathrm{C}$.

\section{qRT-PCR analysis}

Plant leaf samples were ground with liquid nitrogen using a chilled mortar and pestle until they became fine powder. Total RNA was extracted using TRI-Reagent ${ }^{\circ}$ (Molecular Research Center, USA) following the manufacturer's protocol, then the RNA was dissolved in diethylpyrocarbonate-treated water. The quantity and quality of the extracted RNA were checked by a spectrophotometric method and agarose gel electrophoresis. The RNA was treated with RNAse-free DNase I (Thermo Fisher, USA) and converted to cDNA using an iScript $^{\mathrm{tm}} \mathrm{cDNA}$ Synthesis Kit (Bio-Rad, USA). The qRT-PCRs were performed using SsoFast ${ }^{\mathrm{tm}}$ EvaGreen $^{\circ}$ Supermixes (Bio-Rad, USA). The sequences and PCR conditions of the primers used in this study are shown in Additional file 3. Gene expression levels were calculated employing the $2^{-(\Delta \Delta C T)}$ and $2^{-(\Delta C T)}$ methods with $E F 1-\alpha$ as the internal control. For the rice gene 
expression analysis, four biological replicates were used, and for the Arabidopsis gene expression analysis, five biological replicates were used.

\section{Construction of transgenic Arabidopsis expressing OsICL}

Two recombinant plasmids were constructed to express OsICL in Arabidopsis, namely, pAtICL-OsICL-pK2GW7, which was designed to drive OsICL expression using the native AtICL promoter, and p35SCaMV-OsICL-pK2GW7, which was designed to drive OsICL overexpression using the 35 cauliflower mosaic virus (35SCaMV) promoter. To construct those recombinant plasmids, three DNA fragments, namely, $\beta$-glucuronidase and nopaline synthase terminator (GUS-NOS) from pCAMBIA1301, the 2138bp upstream sequence of the Arabidopsis isocitrate lyase gene (pAtICL) from the Arabidopsis genomic DNA, and the rice isocitrate lyase (OsICL) coding sequence from the japonica rice cDNA library (AK063353, KOME clone number 001-114-C03), were amplified by PCR. First, the DNA fragment of the OsICL coding sequence was engineered with an $N d e I$ restriction site on the $5^{\prime}$ end using primers NdeI_OsICL_F and OsICL_R (Additional file 4), before cloning into pTZ57R/T, a TA cloning plasmid (Thermo Fisher, USA), using blue-white colony screening on an ampicillin plate, resulting in OsICL-pTZ57R/T. The $\mathrm{pAtICL}$ sequence was engineered with $\mathrm{Xba \textrm {I }}$ and NdeI restriction sites using primers XbaI_pAtICL_F and pAtICL_NdeI_R (Additional file 4), and cloned into OsICL-pTZ57R/T at the XbaI and NdeI restriction sites. The resulting $\mathrm{p}$ AtICL-OsICL-pTZ57R/T was doubledigested by EcoRI and XbaI. The GUS-NOS fragment was engineered with $E c o R I$ and $X b a I$ restriction sites using primers; EcoRI_DTOPO_GUS-NOS_F and GUSNOS_XbaI_R (Additional file 4), and then cloned into the $\mathrm{pAtICL}$-OsICL-pTZ57R/T, resulting in the recombinant plasmid, GUS-NOS-pAtICL-OsICL-pTZ57R/T. Then, a cassette of GUS-NOS-pAtICL-OsICL was amplified from this recombinant plasmid using DTOPO_ GUS-NOS_F and OsICL_R primers (Additional file 4). The GUS-NOS-pAtICL-OsICL fragment was inserted into a pENTR/D-TOPO gateway directional cloning plasmid (Invitrogen ${ }^{\mathrm{Tw}}$, USA). The cassette of GUS-NOSpAtICL-OsICL in pENTR/D-TOPO was cloned to a pK2GW7 vector by Gateway cloning using LR clonase II (Invitrogen, USA). This recombinant plasmid was designed to express OsICL under the native AtICL promoter. Two other plasmids designed to overexpress OsICL and OsCam1-1 were constructed using the OsICL fragment, which was added with CACC in front of the start codon using primers, DTOPO_OsICL_F and OsICL_R (Additional file 4). The fragment was directionally cloned into a pENTR-DTOPO vector, then further cloned into a pK2GW7 vector by Gateway cloning. Therefore, OsICL should be driven by the 35SCaMV promoter on the $\mathrm{pK} 2 \mathrm{GW} 7$ vector. The DNA sequences of the recombinant plasmids, GUS-NOS-pAtICL-OsICLpK2GW7 (Additional file 5), and OXOsICL-pK2GW7 (Additional file 6) were determined. Agrobacterium tumefaciens GV3101 was transformed with individual plasmids and used for floral dipping [41] of the Aticl Arabidopsis mutant to generate Arabidopsis lines expressing OsICL under a 2138-bp upstream sequence of AtICL (3FL9), and overexpressing OsICL (OXOsICL/ $i c l$ ). A. tumefaciens was also transformed with OXOsICL-pK2GW7 and used for floral dipping of the WT Arabidopsis to generate the OsICL (OXOsICL/WT) overexpressing line.

The floral-dipped Arabidopsis seeds (T1) were selected on $1 \% \mathrm{w} / \mathrm{v}$ sucrose MS medium with $50-100 \mu \mathrm{g} / \mathrm{ml}$ kanamycin. Survival rate of the T2 seeds were determined by germinating $30-100$ seeds from each T1 plant in kanamycin. The expected survival rate of the hemizygous T2, which has no allelic counterpart, was $75 \%$. The $\chi^{2}$ test was applied and statistical significance was set at $p<0.05$. The T2 seeds that exhibited the accepted survival rate of $3: 1$ were grown further to obtain the offspring seeds (T3). The T3 seeds were grown on the MS medium with kanamycin to find homozygous lines among the mixture of hemizygous and homozygous plants. Therefore, survival rate of $100 \%$ was expected for the homozygous plants.

\section{Genotyping of the transgenic Arabidopsis plants by PCR}

Genomic DNA was extracted by grinding plant leaf (around $100 \mathrm{mg}$ ) using a Mixer Mill MM 400 (Retsch, Germany) in a $1.5-\mathrm{ml}$ microtube. Then, $300 \mu \mathrm{l}$ of plant genomic extraction buffer containing $200 \mathrm{mM}$ Tris buffer (pH 7.5), $25 \mathrm{mM}$ EDTA, $250 \mathrm{mM} \mathrm{NaCl}$, and $0.05 \% \mathrm{w} /$ $v$ SDS was mixed with the samples. Equal volume of 99\% isopropanol was added to the sample mixture and mixed. The mixture was centrifuged at $12,000 \times \mathrm{g}$ for 15 min, then the supernatant was discarded. The pellet was dried at room temperature for about $15 \mathrm{~min}$ or until the isopropanol completely evaporated. Then, $50 \mu \mathrm{l}$ of sterilized distill water was added to dissolve the pellet.

The mutant was confirmed by PCR genotyping using three primers, LP, RP, and LB (Additional file 7). LP and $\mathrm{RP}$ were located on the gene and LB was located on the inserted T-DNA on the 4th exon of AtICL (Additional file 8). The PCR product using the LP and RP primers should be about 911 bp in Arabidopsis without the T-DNA insertion (WT), whereas the PCR product using the LB and RP primers should be about $500 \mathrm{bp}$ in Arabidopsis containing T-DNA-inserted AtICL (Aticl mutant). The inserted TDNA in the mutant is sufficiently long to obstruct PCR amplification using the LP and RP primers. In the heterozygous plants, two PCR products of $911 \mathrm{bp}$ and about $500 \mathrm{bp}$ 
should be expected; therefore, one more generation was needed to select homozygous plants to obtain the mutant for further experiments.

PCR genotyping employing specific primer OsICL Seq_M3_F (Additional file 7), which is located on the coding region of OsICL, and 35S_Terminator_Seq_R (Additional file 7) was conducted to confirm OsICL insertion into the transgenic Arabidopsis. The PCR product size of the transgenic Arabidopsis expressing OsICL was expected to be about $460 \mathrm{bp}$.

\section{Isocitrate lyase activity assay}

Approximately $50 \mathrm{mg}$ of total plant leaf tissues from the five Arabidopsis lines, 3FL9, OXOsICL/icl, OXOsICL/ WT, Aticl mutant, and WT, grown in $1 \% \mathrm{w} / \mathrm{v}$ sucrose MS medium for 10 days from three biological replicates were collected, frozen in liquid nitrogen and ground to a fine powder using a Mixer Mill. Then, $500 \mu \mathrm{l}$ of extraction buffer $(100 \mathrm{mM}$ potassium phosphate buffer at $\mathrm{pH}$ 7.6, $10 \mathrm{mM} \mathrm{MgCl}_{2}, 1 \mathrm{mM}$ EDTA, and $1 \mathrm{mM} \mathrm{DTT)} \mathrm{[22]}$ was added to the ground samples and mixed by vortex. The mixture was centrifuged at $12,000 \times \mathrm{g}$ for $20 \mathrm{~min}$ at $4{ }^{\circ} \mathrm{C}$. The supernatant was transferred to a new microtube for use as crude protein extract in the enzymatic activity assay and the pellet was discarded.

Next, $50 \mu \mathrm{l}$ of extracted protein was added into $440 \mu \mathrm{l}$ of reaction buffer containing $50 \mathrm{mM}$ potassium phosphate buffer at pH 6.9, $50 \mathrm{mM} \mathrm{MgCl} 2,10 \mathrm{mM}$ EDTA, and $40 \mathrm{mM}$ phenylhydrazine, which was modified from Cooper and Beevers [15], then mixed at $30^{\circ} \mathrm{C}$ using a pipette in a $1-\mathrm{ml}$ quart cuvette with $1-\mathrm{cm}$ path length. The absorbance of the reaction mixture at $\mathrm{A}_{324}$ was measured by spectrophotometer until it was stable, then $10 \mu \mathrm{l}$ of $500 \mathrm{mM} \mathrm{D}-\mathrm{L}$ isocitric acid was added to the mixture and homogeneously mixed. Then, the absorbance of the reaction mixture at $\mathrm{A}_{324}$ was measured for $10 \mathrm{~min}$. The difference between the $0 \mathrm{~min}$ and $10 \mathrm{~min}$ $\mathrm{A}_{324}$ values was used to calculate the amount of glyoxylate production per $10 \mathrm{~min}$ using the glyoxylate standard curve. The protein content of an aliquot of the crude protein extract was determined using Bradford reagent in a 96-well plate. Absorbance at $A_{595}$ was measured using a microplate reader Synergy H1 (Biotek ${ }^{\circ}$, USA), and bovine serum albumin was used to construct a standard curve.

\section{Growth measurements}

Seeds of the five Arabidopsis lines; 3FL9, OXOsICL/icl, OXOsICL/WT, Aticl mutant and WT were decontaminated as described above. Then, the seeds were orderly sown onto MS medium with $0,100,120,150$, or 200 $\mathrm{mM} \mathrm{NaCl}$. The Arabidopsis plates were stored at $4{ }^{\circ} \mathrm{C}$ for 2 days. The number of germinated seeds was observed daily for 7 days after the plates were moved to the growing condition. We performed five biological replicates, and each biological replicate contained 50 seeds of each line. The germination rate was reported as percentage with standard deviation.

Following these plant growing steps, the growing period was extended to 10 days. Then, 10 seedlings of each biological replicate of the Aticl mutant and WT Arabidopsis, 3FL9, OXOsICL/icl, and OXOsICL/WT were weighed to determine the fresh weight. The samples were then baked at $60-70{ }^{\circ} \mathrm{C}$ in a hot air oven for 5-7 days. The baked samples were weighed to determine the dry weight.

\section{Leaf $F_{v} / F_{m}$ and temperature measurement}

The 4-week-old vegetative stage of the five Arabidopsis lines, 3FL9, OXOsICL/icl, OXOsICL/WT, Aticl mutant, and WT, growing in pots containing 3:1:1 of peat moss: perlite:vermiculite were treated with $300 \mathrm{mM} \mathrm{NaCl}$ for 3 days. Then dark adaptation was allowed for 30 mins before the $F_{\mathrm{v}} / F_{\mathrm{m}}$ was measured under the dark condition using a pocket PEA fluorometer (Hansatech, UK). Rosette leaf temperature was measured using a FLIR C2 thermal camera (FLIR, USA). For $F_{\mathrm{v}} / F_{\mathrm{m}}$, four biological replicates were used, and for leaf temperature, six biological replicates were used.

\section{Sugar content measurements}

The 4-week-old vegetative stage of the five Arabidopsis lines, 3FL9, OXOsICL/icl, OXOsICL/WT, Aticl mutant, and WT, growing in pots containing 3:1:1 of peat moss: perlite:vermiculite from six biological replicates were treated with $300 \mathrm{mM} \mathrm{NaCl}$ for 3 days. The rosette leaves used were collected, frozen in the liquid nitrogen, and lyophilized. The dry weight was measured and the samples were ground to fine powder. The samples were extracted in deionized water and filtered. Glucose, sucrose and fructose contents were determined using high performance liquid chromatography (HPLC) (Shimadzu, Japan) with Hi-Plex Ca (Duo) column (Agilent, USA). The HPLC flow rate was $0.25 \mathrm{ml} / \mathrm{min}$, column temperature was $85^{\circ} \mathrm{C}, 100 \%$ ultra-pure water was used as the mobile phase, and a refractive index detector (RID) was used to detect the sugars.

\section{Statistical analysis}

Data from q RT-PCRs, enzyme activity assays, and growth parameters were compared using analysis of variance (ANOVA), and the means were compared with Duncan's multiple range test, with significance set as $p<0.05$. The germination rate data were compared using ANOVA, and the means were compared with Duncan's multiple range test, with significance set as $p<0.01$. 


\section{Supplementary information}

Supplementary information accompanies this paper at https://doi.org/10. 1186/s12870-019-2086-2

Additional file 1. A) PCR amplification verifying the cloning of GUS-NOS, AtICL upstream sequence, and Os/CL coding sequence into the recombinant plasmid GUS-NOS-upstream-At/CL-Os/CL-pK2GW7: lane M, DNA marker; lane 1, PCR product of the cloned GUS-NOS with size of around $2300 \mathrm{bp}$; lane 2, PCR product of the cloned At/CL upstream sequence with size of around $2100 \mathrm{bp}$; lane 3, PCR product of the cloned Os/CL coding sequence with size of around $1700 \mathrm{bp}$. B) PCR amplification verifying the insertion of OSICL coding sequence in the recombinant plasmid Os/CL-pK2GW7: lane M, DNA marker; lane 1, PCR product of the cloned Os/CL coding sequence with size of around $1700 \mathrm{bp}$. C) PCR genotyping of the transgenic Arabidopsis background: lane M, DNA marker; lane 1 aticl mutant; lane 2 wild type; lane 3 3FL9; lane 4 OXOs/CL/ icl; lane 5 OXOs/CLMT. D) PCR amplification verifying OS/CL gene insertion: lane M, DNA marker; lane 1 aticl mutant; lane 2 wild type; lane 3 3FL9; lane 4 OXOS/CL/icl; lane 5 OXOs/CLMT. The PCR products were analyzed by agarose gel electrophoresis using TAE buffer with $1 \%$ agarose gel under $100 \mathrm{mV}$ for $30 \mathrm{~min}$

Additional file 2. Thermograms of the five Arabidopsis lines from FLIR C2 thermal camera.

Additional file 3. Nucleotide sequences and PCR conditions of the primers used for qRT-PCR.

Additional file 4. Nucleotide sequences of the primers used for construction of the recombinant plasmids for expressing Os/CL. The bold characters represent restriction sites, the italic characters represent the added nucleotides benefitting in binding of restriction enzyme, and the underlined characters represent the sequence benefitting in directional TOPO cloning.

Additional file 5. Schematic diagrams showing construction of the recombinant plasmid for expressing Os/CL under the control of $A t / C L$ promoter. A) the three fragments were inserted into pTZ57R/T employing restriction site cloning strategy, and the target fragments were amplified from the recombinant plasmid GUS-NOS-pAt/CL-Os/CL-pTZ57R/T by PCR. B) The GUS-NOS-pAt/CL-Os/CL cassette was directionally inserted into pENTR/DTOPO employing "CACC" site, then it was subcloned into pK2GW7, the destination vector, by Gateway cloning strategy.

Additional file 6. Schematic diagrams showing construction of the recombinant plasmid for overexpressing OSICL employing directional TOPO and Gateway cloning strategies.

Additional file 7. Nucleotide sequences of the primers used for PCR genotyping the wild type, the aticl mutant, and the transgenic Arabidopsis lines.

Additional file 8. The inserted T-DNA location on the icl Arabidopsis mutant (GK-008E03) and the positions of the primers for icl Arabidopsis mutant genotyping: LP and RP represent primers locating on the At/CL gene and LB represents primer locating on the inserted T-DNA.

\section{Abbreviations}

ABA: Abscisic acid; AtICL: Arabidopsis thaliana isocitrate lyase gene; CaM: Calmodulin; ICL: Isocitrate lyase; OsCam 1-1: Oryza sativa L. calmodulin 1-1 gene; Os/CL: Oryza sativa L. isocitrate lyase gene; TCA: Tricarboxylic acid cycle; WT: Wild type

\section{Acknowledgements}

We thank Thammaporn Kojonna for their assistance with the $F_{v} / F_{m}$ measurements. We thank Margaret Biswas, PhD, from Edanz Group (www. edanzediting.com/ac) for critically reviewing and editing a draft of this manuscript.

\section{Authors' contributions}

WY carried out the laboratory work in collaboration with SS and LQ, WY and TB performed the data analysis and interpretation. WY prepared the figures and tables. WY and TB drafted the manuscript. All authors read and approved the final manuscript.

\section{Funding}

This work was supported partially by the Thailand Research Fund (No. BRG5680019) to T.B. and by the Chulalongkorn University Fund (Ratchadaphiseksompot Endowment Fund). W.Y. was supported by the Royal Golden Jubilee Ph.D. Program-RGJ (PHD/0043/2556-4.C.CU/56/G.1.O.XX) from the Thailand Research Fund. Omics Sciences and Bioinformatics Center was supported under the Outstanding Research Performance Program: Chulalongkorn Academic Advancement into Its second Century Project (CUAASC). Each of the funding bodies granted the funds based on research proposals and had no influence on the experimental design, data collection, analysis and interpretation, or on writing the manuscript.

\section{Availability of data and materials}

All relevant data are included in this article and its Additional files.

\section{Ethics approval and consent to participate}

Not applicable.

\section{Consent for publication}

Not applicable.

\section{Competing interests}

The authors declare that they have no competing interests.

\section{Author details}

${ }^{1}$ Molecular Crop Research Unit, Department of Biochemistry, Faculty of Science, Chulalongkorn University, Bangkok 10330, Thailand. ${ }^{2}$ Omics Sciences and Bioinformatics Center, Faculty of Science, Chulalongkorn University, Bangkok 10330, Thailand. 'State Key Laboratory for Protein and Plant Gene Research, Peking-Tsinghua Center for Life Sciences at College of Life Sciences, Peking University, Beijing 100871, China. ${ }^{4}$ The National Plant Gene Research Center (Beijing), Beijing 100101, China.

Received: 6 June 2019 Accepted: 20 October 2019

Published online: 06 November 2019

\section{References}

1. Chaves MM, Flexas J, Pinheiro C. Photosynthesis under drought and salt stress: regulation mechanisms from whole plant to cell. Ann Bot. 2009; 103(4):551-60

2. Zeng L, Shannon MC, Lesch SM. Timing of salinity stress affects rice growth and yield components. Agr Water Manage. 2001;48(3):191-206.

3. Horie T, Karahara I, Katsuhara M. Salinity tolerance mechanisms in glycophytes: an overview with the central focus on rice plants. Rice. 2012;5(1):11.

4. Munns R, Day DA, Fricke W, Watt M, Arsova B, Barkla BJ, Bose J, Byrt CS, Chen Z-H, Foster KJ, et al. Energy costs of salt tolerance in crop plants. New Phytol. 2019;0(0). in press

5. Knight H. Calcium signaling during abiotic stress in plants. In: International review of cytology - a survey of cell biology, vol. 195; 1999. p. 269-324.

6. Tuteja N, Mahajan S. Calcium signaling network in plants: an overview. Plant Signal Behav. 2007;2(2):79-85.

7. Einspahr KJ, Thompson GA. Transmembrane signaling via phosphatidylinositol 4,5-bisphosphate hydrolysis in plants. Plant Physiol. 1990;93(2):361.

8. Mahajan S, Tuteja N. Cold, salinity and drought stresses: an overview. Arch Biochem Biophys. 2005:444(2):139-58

9. Ranty B, Aldon D, Cotelle V, Galaud J-P, Thuleau P, Mazars C. Calcium sensors as key hubs in plant responses to biotic and abiotic stresses. Front Plant Sci. 2016;7:327.

10. Yuenyong W, Chinpongpanich A, Comai L, Chadchawan S, Buaboocha T. Downstream components of the calmodulin signaling pathway in the rice salt stress response revealed by transcriptome profiling and target identification. BMC Plant Biol. 2018;18(1):335

11. Cornah JE, Germain V, Ward JL, Beale MH, Smith SM. Lipid utilization, gluconeogenesis, and seedling growth in Arabidopsis mutants lacking the glyoxylate cycle enzyme malate synthase. J Biol Chem. 2004;279(41): 42916-23.

12. Eastmond PJ, Germain V, Lange PR, Bryce JH, Smith SM, Graham IA. Postgerminative growth and lipid catabolism in oilseeds lacking the gloxylate cycle. P Natl Acad Sci USA. 2000;97(10):5669-74. 
13. Lu Y, Wu Y-R, Han B. Anaerobic induction of isocitrate lyase and malate synthase in submerged rice seedlings indicates the important metabolic role of the glyoxylate cycle. Acta Bioch Bioph Sin. 2005;37(6):406-14.

14. Thorneycroft $D$, Sherson SM, Smith SM. Using gene knockouts to investigate plant metabolism. J Exp Bot. 2001;52(361):1593-601.

15. Cooper TG, Beevers H. Mitochondria and glyoxysomes from castor bean endosperm: enzyme constituents and catalytic capacity. J Biol Chem. 1969; 244(13):3507-13.

16. Nishimura M, De Bellis L. Development of enzymes of the glyoxylate cycle during senescence of pumpkin cotyledons. Plant Cell Physiol. 1991;32(4):555-61.

17. Olsson P, Yilmaz JL, Sommarin M, Persson S, Bülow L. Expression of bovine calmodulin in tobacco plants confers faster germination on saline media. Plant Sci. 2004;166(6):1595-604

18. Saeng-ngam S, Takpirom W, Buaboocha T, Chadchawan S. The role of the OsCam1-1 salt stress sensor in ABA accumulation and salt tolerance in rice. J Plant Biol. 2012;55(3):198-208.

19. Goodstein DM, Shu S, Howson R, Neupane R, Hayes RD, Fazo J, Mitros T, Dirks W, Hellsten U, Putnam N, et al. Phytozome: a comparative platform for green plant genomics. Nucleic Acids Res. 2012;40(D1):D1178-86.

20. Higo K, Ugawa Y, Iwamoto M, Korenaga T. Plant cis-acting regulatory DNA elements (PLACE) database: 1999. Nucleic Acids Res. 1999;27(1):297-300.

21. Oxborough $\mathrm{K}$, Baker NR. Resolving chlorophyll a fluorescence images of photosynthetic efficiency into photochemical and non-photochemical components - calculation of $\mathrm{qP}$ and Fv-/Fm-; without measuring Fo. Photosynth Res. 1997:54(2):135-42.

22. Sidari M, Mallamaci C, Muscolo A. Drought, salinity and heat differently affect seed germination of Pinus pinea. J Forest Res-Jpn. 2008;13(5):326-30.

23. Huang Y-J, To K-Y, Yap M-N, Chiang W-J, Suen D-F, Chen S-CG. Cloning and characterization of leaf senescence up-regulated genes in sweet potato. Physiol Plantarum. 2001;113(3):384-91

24. Lee $\mathrm{RH}$, Wang $\mathrm{CH}$, Huang LT, Chen SCG. Leaf senescence in rice plants: cloning and characterization of senescence up-regulated genes. J Exp Bot. 2001;52(358):1117-21.

25. Gut H, Matile P. Apparent induction of key enzymes of the glyoxylic acid cycle in senescent barley leaves. Planta. 1988;176(4):548-50.

26. McLaughlin JC, Smith SM. Metabolic regulation of glyoxylate-cycle enzyme synthesis in detached cucumber cotyledons and protoplasts. Planta. 1994 195(1):22-8

27. Chen Z, Walker R, Acheson R, Técsi LI, Wingler A, Lea P, Leegood R. Are isocitrate lyase and phosphoenolpyruvate carboxykinase involved in gluconeogenesis during senescence of barley and cucumber cotyledons? Plant Cell Physiol. 2000;41:960-7.

28. Munns R. Comparative physiology of salt and water stress. Plant Cell Environ. 2002;25(2):239-50.

29. Lutts S, Kinet JM, Bouharmont J. NaCl-induced senescence in leaves of rice (Oryza sativa L.) cultivars differing in salinity resistance. Ann Bot. 1996;78(3): 389-98.

30. Dai C, Lee Y, Lee IC, Nam HG, Kwak JM. Calmodulin 1 regulates senescence and ABA response in Arabidopsis. Front Plant Sci. 2018;9:803.

31. Wilkinson S, Davies WJ. ABA-based chemical signalling: the co-ordination of responses to stress in plants. Plant Cell Environ. 2002;25(2):195-210.

32. Tuteja N. Abscisic acid and abiotic stress signaling. Plant Signal Behav. 2007; 2(3):135-8.

33. Mao C, Lu S, Lv B, Zhang B, Shen J, He J, Luo L, Xi D, Chen X, Ming F. A rice NAC transcription factor promotes leaf senescence via ABA biosynthesis. Plant Physiol. 2017;174(3):1747

34. Xie Z, Zhang Z-L, Zou X, Huang J, Ruas P, Thompson D, Shen QJ. Annotations and functional analyses of the rice WRKY gene superfamily reveal positive and negative regulators of abscisic acid signaling in aleurone cells. Plant Physiol. 2005;137(1):176.

35. Li Y, Beisson F, Pollard M, Ohlrogge J. Oil content of Arabidopsis seeds: the influence of seed anatomy, light and plant-to-plant variation. Phytochemistry. 2006;67(9):904-15.

36. de los Reyes BG, Myers SJ, McGrath JM. Differential induction of glyoxylate cycle enzymes by stress as a marker for seedling vigor in sugar beet (Beta vulgaris). Mol Gen Genomics. 2003;269(5):692-8.

37. Zamin SS, Jung-\|I C, Sung-Han P, Taek-Ryoun K, Gang-Seob L, Mi-Jeong J, Kyung-Whan K, Seong-Kon L, Soo-Chul P. Phenotyping of rice in salt stress environment using high-throughput infrared imaging. Acta Bot Croat. 2014; 73(1):312-21.
38. Hildebrandt Tatjana M, Nunes Nesi A, Araújo Wagner L, Braun H-P. Amino acid catabolism in plants. Mol Plant. 2015:8(11):1563-79.

39. Che-Othman MH, Jacoby RP, Millar AH, Taylor NL. Wheat mitochondrial respiration shifts from the tricarboxylic acid cycle to the GABA shunt under salt stress. New Phytol. 2019;0(0). in press

40. Yoshida S, Forno AD, Cock HJ, Gomez AK. Laboratory manual for physiological studies of rice. Manila: International Rice Research Institute 1976.

41. Clough SJ, Bent AF. Floral dip: a simplified method for agrobacterium -mediated transformation of Arabidopsis thaliana. Plant J. 1998;16(6):735-43.

\section{Publisher's Note}

Springer Nature remains neutral with regard to jurisdictional claims in published maps and institutional affiliations.
Ready to submit your research? Choose BMC and benefit from:

- fast, convenient online submission

- thorough peer review by experienced researchers in your field

- rapid publication on acceptance

- support for research data, including large and complex data types

- gold Open Access which fosters wider collaboration and increased citations

- maximum visibility for your research: over $100 \mathrm{M}$ website views per year

At $\mathrm{BMC}$, research is always in progress.

Learn more biomedcentral.com/submissions 